\section{SPINAL CORD INJURY}

\section{Spinal stimulation steps up}

Capogrosso, M. et al. Nat Protoc 13, 2031-2061 (2018)
An effective treatment strategy for spinal cord injuries is a holy grail of rehabilitation medicine. So far, however, researchers have had little success in repairing the spinal lesion itself, and the strategy of bypassing the spine entirely by electrically stimulating muscle has also yielded disappointing results. More recently, a technique called epidural electrical stimulation (EES) of the spinal cord has emerged as a promising alternative in animal models, and, preliminary results suggest, in humans.

EES involves stimulating the dorsal roots of the spinal cord from which motor neurons innervate specific muscles. In 2016, researchers led by Grégoire Courtine at the Swiss Federal Institute of Technology published two papers showing that electrical stimulation of the lumbar spine could restore locomotion in rats (Cell 89, P814-828; 2016), a traditional animal model of spinal cord injuries, as well as in nonhuman primates (Nature 539, 284-288; 2016). "This was the first demonstration that this kind of technology can work for locomotion in something that is not a rat," says Marco Capogrosso, a biomedical engineer at the University of Fribourg and first author on the latter paper.

In a recent report in Nature Protocols, Capogrosso, Courtine and their colleagues present the experimental protocol for those studies in great detail. They describe the surgeries for implanting the stimulator, the anatomy underlying how to position the stimulating electrodes, and procedure for designing stimulation protocols appropriately timed to generate locomotion. "As the field works to translate the results of studies like the [2016] Capogrosso paper into human clinical translational work, having such a comprehensive reference is extremely valuable," says Douglas Weber, a neuroscientist and bioengineer at the University of Pittsburgh who was not involved in the work. "I think it's a roadmap for what might be possible in humans in the next five or 10 years."

EES targets specific dorsal roots that drive stimulation of specific muscles, so the stimulator must be a multi-electrode array in which the electrodes are spaced to conform to the anatomy of the spinal cord. That means that unlike functional electrical stimulation of muscles, just a few correctly placed electrodes can generate the movement.

The real crux of the technique, though, lies in matching the pattern and timing of the external stimulation to the specific phases that make up an animal's gait as the

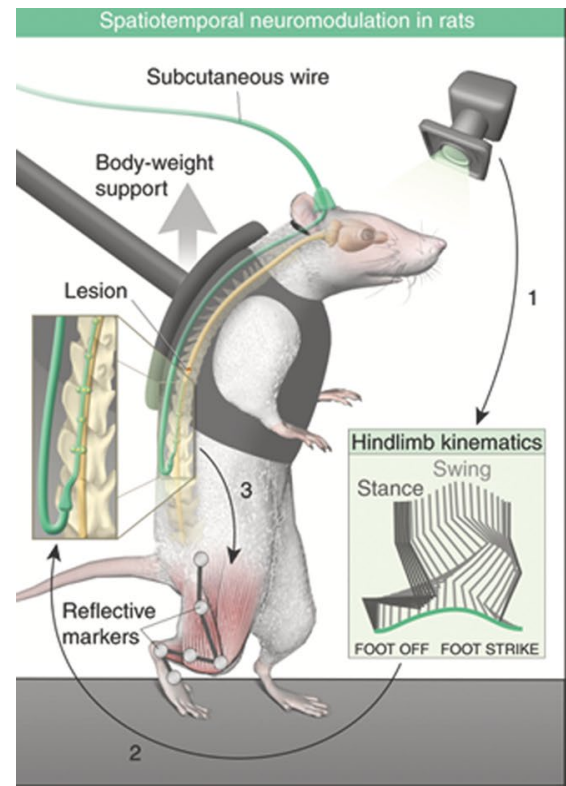

(1) A motion capture system monitors the foot trajectory.

(2) The kinematics of attempted movements is used to detect gait events, which trigger spinal cord stimulation.

(3) Spinal cord stimulation promotes flexion and extension of the leg and enables walking.

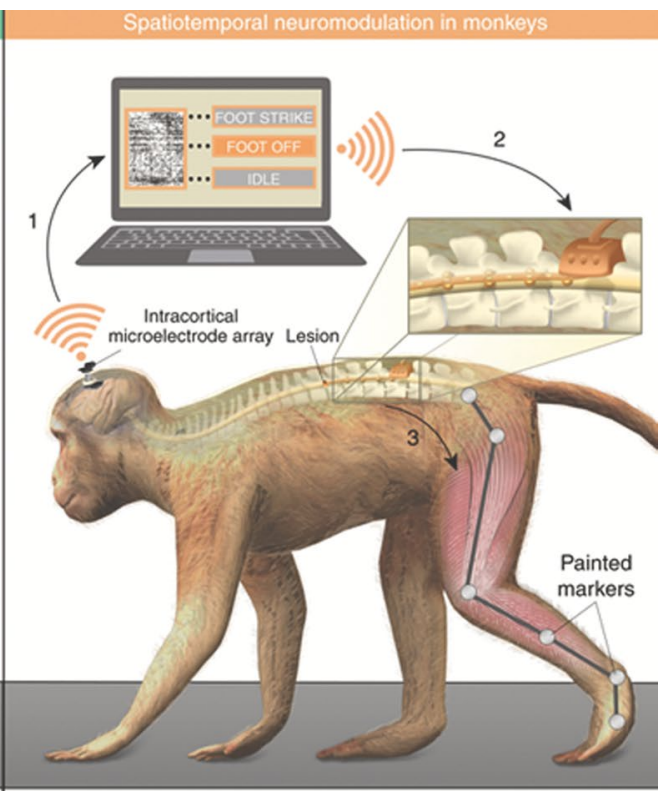

(1) A microelectrode array transmits motor cortical activity to a computer.

(2) Motor cortical activity is used to predict gait events, which trigger spinal cord stimulation wirelessly.

(3) Spinal cord stimulation promotes flexion and extension of the leg and enables walking.

Rat and nonhuman primate spinal cord neuromodulation. Credit: Reprinted with permission from Capogrosso et al. 2018 (Springer Nature)

animal moves in real time. For example, a gait cycle that involves the extension and flexion of the leg has two phases that the authors define as the "foot off" and the "foot strike" events; specific spinal segments must be stimulated at distinct points in this cycle. "This is a hypothesis-driven technology" based on years of studying the anatomy and electrophysiology of the spinal cord, Capogrosso says. "The tuning of the system is not a trial and error thing."

In animals that still have partial movement, gait kinematics can be determined via sensors or biometric signals such as electrophysiology of the muscles. In fully paralyzed animals, this information must be obtained from the brain signals that encode the movement. A real-time control system then uses this kinematic information to deliver bursts of stimulation during the right phase of the gait cycle to produce a coordinated movement. The movement won't precisely match the gait cycle of a healthy animal, but it does move the needle from complete paralysis to coordinated locomotion. "What's elegant about this approach is that it's leveraging the residual or spared circuitry in the spinal cord to generate movements in a near-naturalistic fashion," says Weber.

The technique is ripe for translation, Capogrosso says. In fact, Courtine is conducting a first-in-man clinical trial of the technology in patients with incomplete spinal cord injuries. Capogrosso, meanwhile, is testing an adapted version of the technology on upper limb paralysis in primates. However, the hardware needs some improvements. For example, the only spinal implant on the market was designed for another purpose-treating chronic painand is the wrong size to selectively target the human dorsal roots, Capogrosso says. The stimulator also needs an overhaul. "Ideally we would want a stimulator that is much faster and that can change the currents it delivers in real time," he explains. These technologies must be commercially developed, he says. “That's why our preclinical results are so important-they show that the industry needs to get to work in this direction."

\section{Alla Katsnelson}

Published online: 23 October 2018 https://doi.org/10.1038/s41684-018-0181-X 